\title{
Blind statistical indicators of the kinetic Sunyaev-Zel'dovich anisotropies
}

\author{
M. Sorel, N. Aghanim, and O. Forni \\ IAS-CNRS, Université Paris Sud, Bâtiment 121, 91405 Orsay Cedex, France \\ Received 9 July 2002 / Accepted 30 August 2002

\begin{abstract}
We report on a study aiming at characterising the kinetic Sunyaev-Zel'dovich (KSZ) effect through statistical criteria that can blindly indicate the presence of secondary anisotropies due to KSZ effect buried in the Cosmic Microwave Background (CMB) anisotropies. We show, using simulated maps, that some properties of the covariance coefficient between thermal Sunyaev-Zel'dovich (TSZ) and the temperature fluctuation map can be used in that purpose.
\end{abstract}

Key words. cosmology: cosmic microwave background - methods: statistical

\section{Introduction}

The Cosmic Microwave Background (CMB) anisotropies represent a superposition of temperature fluctuations that are either generated before recombination, the so-called primary anisotropies, or after recombination, (they are called secondary in this case). The Sunyaev-Zel'dovich (SZ) effect (Sunyaev \& Zel'dovich 1980) (or Birkinshaw 1999 for a recent review) induces secondary anisotropies through the interaction of CMB photons with the free electrons of the ionised and hot gas in galaxy clusters. The inverse Compton interaction between photons and hot electrons globally at rest with respect to each other induces a second order effect (in $v_{\mathrm{e}} / c$ ) called the thermal SZ effect (TSZ). It is characterised by a peculiar spectral signature with brightness decrement and increment respectively around $2 \mathrm{~mm}$ and $0.8 \mathrm{~mm}$, and a null effect around $1.4 \mathrm{~mm}$. The Doppler effect, induced when the galaxy cluster moves with respect to the CMB rest frame, generates first order (in $v_{\mathrm{e}} / c$ ) secondary anisotropies. They have in this case the same spectral signature as the primary temperature fluctuations. The Doppler effect is commonly known as the kinetic SZ effect (KSZ).

The CMB analysis is becoming one of the most powerful tools for observational cosmology. The primary anisotropies are indeed used to probe the early universe and the cosmological parameters (e.g. Wang et al. 2002; de Bernardis et al. 2002; Pryke et al. 2002). To achieve this goal, the primary anisotropies need to be first separated from the secondary fluctuations. The distinction between the anisotropies due to TSZ effect and the primary anisotropies (and henceforth their separation) is in principle feasible. It takes advantage of the peculiar spectral signature of the TSZ and can thus be achieved through multi-wavelength observations (Hobson et al. 1998;

Send offprint requests to: M. Sorel, e-mail: Maud.Sorel@ias.u-psud.fr
Bouchet \& Gispert 1999; Snoussi et al. 2001). On the contrary, the separation between the primary fluctuations and those produced by the KSZ effect is a priori impossible by means of multi-wavelength observations only, since both have the same spectral signature. The non-Gaussian signature associated with the KSZ anisotropies may be regarded as a way of differentiating the secondary and primary fluctuations. This signature can be measured, in the direct and dual spaces (Fourier, wavelet), through the high order moments, the bispectrum, or the trispectrum. This statistical differentiation is promising. However, it relies on the fact that the primary anisotropies are a priori Gaussian distributed. It also neglects the fact that other sources of non-Gaussian signatures could contribute to the measurement (other secondary anisotropies, e.g. inhomogeneous reionisation (Aghanim \& Forni 1999), galactic emission (Jewell 2001), systematic effects,...). The problem of separating between CMB and KSZ remains therefore mostly unsolved.

In the present study, we will investigate statistical criteria that might allow to indicate in a signal constituted of a mixture of the primary and KSZ secondary anisotropies the contribution from KSZ effect, and thus ease the separation between the two signals.

\section{Analysis and results}

We adopt an empirical approach for the statistical characterisation of the different astrophysical signals and we choose to address the problem through the analysis of simulated maps (15 maps of $512 \times 512$ pixels (12.5 degrees aside)). The major advantage of this choice resides in the fact that the analysis mimics the kind of analysis we can perform on "real" data. Also in this spirit, we always focus on the two observable quantities that we will possibly obtain from multi-wavelength observations: the TSZ map on one hand and, on the other hand, 
a temperature fluctuation map accounting for the nonseparable KSZ and primary CMB anisotropies. We use simulated maps of the TSZ and KSZ effect, and of the primary anisotropies (a description of the simulations can be found in Aghanim et al. 2001). We illustrate our results in the case of the low matter density flat universe with parameters $\Omega_{\mathrm{m}}=0.3$, $\Omega_{\Lambda}=0.7, h=H_{0} / 100 \mathrm{~km} \mathrm{~s}^{-1} \mathrm{Mpc}^{-1}=0.65$. In the following, the temperature fluctuation map (primary $\mathrm{CMB}+\mathrm{sec}-$ ondary KSZ) will be referred to as $\mathrm{KSZ}+\mathrm{CMB}$ map, and the TSZ effect will be expressed in terms of the Compton parameter $(y)$ map.

\subsection{Amplitude of the covariance coefficient}

The interaction of CMB photons with the electrons of the hot ionised gas in galaxy clusters induces both temperature fluctuations and Compton distortions through respectively the KSZ and TSZ effects. Obviously, the maps of the two effects are very closely related. We investigate this property and the way it is affected by the mixing of primary and secondary fluctuations. We first simply investigate the correlation between two quantities TSZ, on the one hand, and either KSZ or KSZ+CMB, on the other hand. We find it a rather limited tool to exhibit the KSZ temperature anisotropies even if considering the correlation with a pure KSZ map. This is due to the fact that the correlation coefficient is a normalised quantity unable to differentiate between large and small signals. We know that large TSZ distortions are not systematically associated with large KSZ anisotropies. We therefore favoured the use of the covariance which is a better-suited tool as it is sensitive to the relative amplitude of the compared signals. The covariance coefficient between two signals $a$ and $b$ is given by:

$C_{\mathrm{cov}}(a, b)=\frac{1}{N} \sum_{i=0}^{N-1}\left(a_{i}-\bar{a}\right)\left(b_{i}-\bar{b}\right)$,

where $\bar{a}$ and $\bar{b}$ stand for the means of $a$ and $b$, and $N$ is the number of elements.

Using Eq. (1), we produce a covariance map by computing the covariance factor, in a $2 \times 2$ pixel window, between the signal $a$ (always TSZ map in our case) and the signal $b$ (KSZ, pure $\mathrm{CMB}$ or $\mathrm{KSZ}+\mathrm{CMB}$ map). The size of the computation window ( 3 arcmin aside) is particularly sensitive to the central region of the clusters.

A simple cut, at the same position, across the simulated maps of the astrophysical processes is displayed, in Fig. 1, upper panel. It is compared, Fig. 1 lower panel, to the cuts (again at the same position) across the covariance maps between TSZ and KSZ (dashed line), TSZ and CMB (thick solid line) and finally TSZ and KSZ+CMB (solid line). A direct comparison between the cuts, shows the following: there are small amplitude covariance coefficients between TSZ and pure CMB. These amplitudes are compatible with only fortuitous and random correlations. The absence of significant correlation between the two signals is not surprising since they are not causally related. The $C_{\text {cov }}(\mathrm{TSZ}, \mathrm{KSZ}+\mathrm{CMB})$ have, on the contrary, significantly larger amplitudes. In addition, large covariance coefficients between TSZ and $\mathrm{KSZ}+\mathrm{CMB}$ are generally
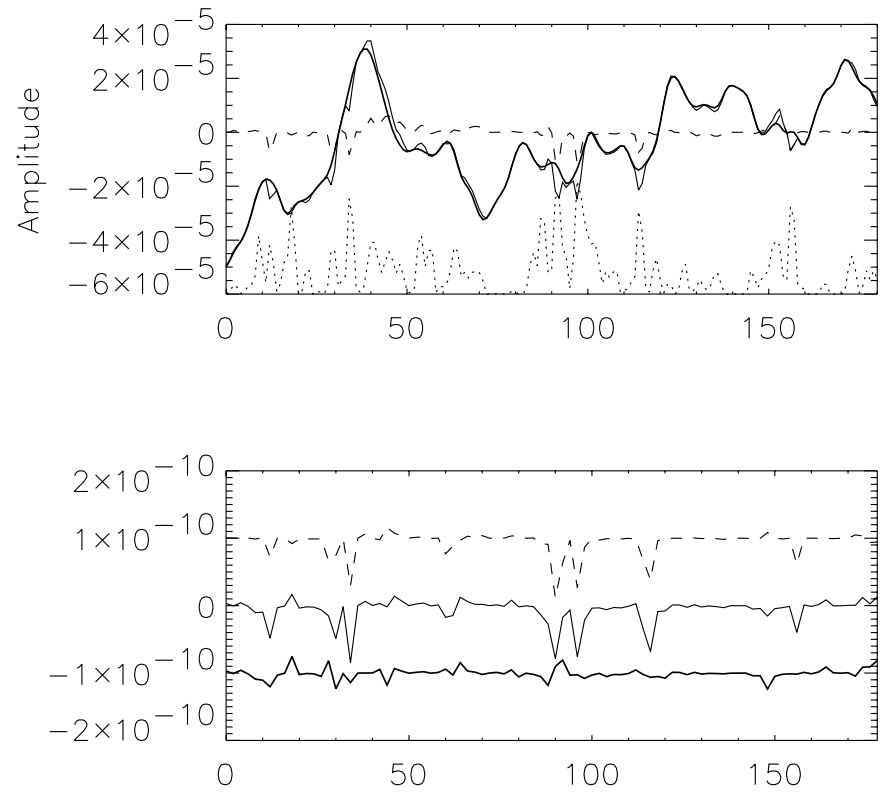

Fig. 1. Upper panel: cuts across the simulated maps. Solid thick line stands for the pure CMB map, dashed line represents the pure KSZ signal, and thin solid line is for the KSZ + CMB. Also displayed in dotted line is the TSZ signal in terms of its $y$ parameter shifted by $-6 \times$ $10^{-5}$. Lower panel: cuts (at the same position) across the covariance maps between TSZ and KSZ (dashed line), TSZ and CMB (thick solid line), and TSZ and KSZ + CMB (solid line). The curves are shifted to ease the reading. Note the similarity between the two upper lines. The covariance coefficients are computed in a $2 \times 2$ pixel window.

associated with the same features in the TSZ and KSZ covariance map. In these cases, the amplitudes of the covariance are almost identical. Moreover, the covariance coefficients behave like the KSZ signal: each of the significantly large covariance coefficient is indeed associated with a temperature fluctuation due to the KSZ effect (Fig. 1, upper panel). This correspondence seems a powerful indicator (both in terms of position and amplitude) of the presence of KSZ fluctuations, and hence galaxy clusters buried in the CMB signal, as it seems to overcome the fact that KSZ and CMB fluctuations have the same spectral signature.

We choose not to address source detection issues suggested by the spatial concordance between the covariance coefficients and the KSZ anisotropies (or in other words the galaxy clusters). We rather focus on a statistical criterion to indicate the presence of KSZ fluctuations that is based on the relation between the amplitudes of the covariance coefficients and the temperature fluctuations.

Instead of investigating the cuts one by one, we use a two dimensional representation in the $\left(C_{\text {cov }},(\delta T / T)_{\mathrm{KSz}}\right)$ plane. This representation is used to investigate how closely the amplitude of the KSZ effect is (or is not) related to the covariance coefficient. As a first step, we plot the covariance coefficients $C_{\text {cov }}$ (TSZ, CMB), between TSZ and the CMB temperature fluctuations $(\delta T / T)_{\mathrm{CMB}}$, as a function of the temperature fluctuations due to KSZ effect $(\delta T / T)_{\mathrm{KSZ}}$. As the two signals are independent, we find that the data points are randomly distributed in the $\left(C_{\mathrm{cov}},(\delta T / T)_{\mathrm{KSZ}}\right)$ plane as displayed in Fig. 2. The absence of a general trend confirms the absence of any physical 


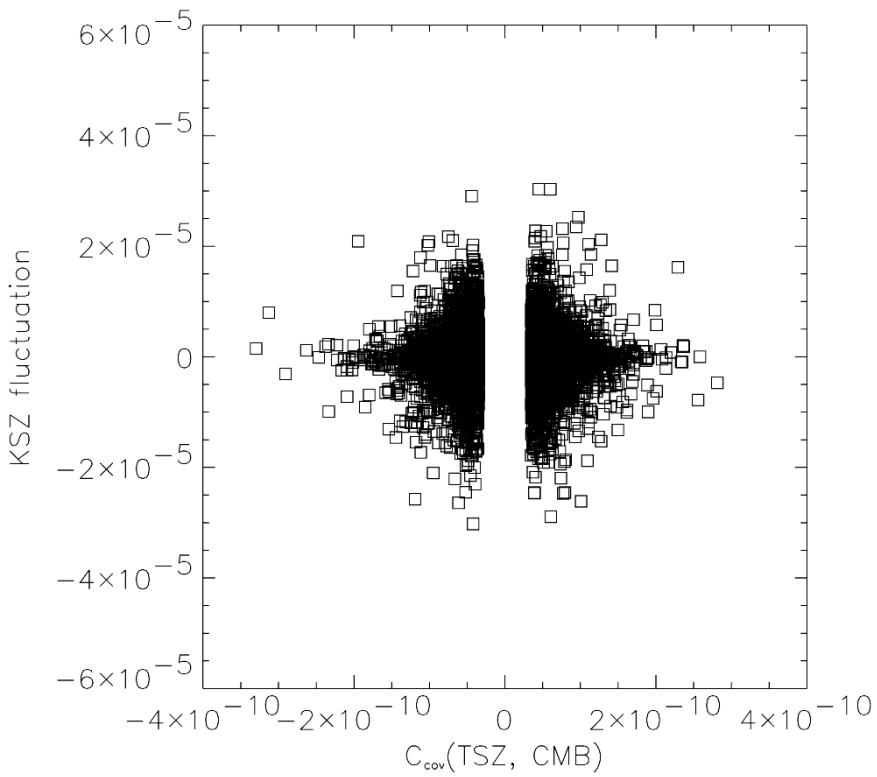

Fig. 2. For the 15 simulated maps, covariance coefficients (above a $3 \sigma$ threshold) computed in a $2 \times 2$ pixel window between the TSZ and primary CMB signals as a function of temperature fluctuation $(\delta T / T)_{\mathrm{KsZ}}$.

relation between the primary $\mathrm{CMB}$ fluctuations and those associated with the SZ effect, and consequently with the galaxy clusters.

On the contrary, when we compute the map of covariance between TSZ and pure KSZ signals, and plot $C_{\text {cov }}(\mathrm{TSZ}, \mathrm{KSZ})$ as a function of $(\delta T / T)_{\mathrm{KSZ}}$ (Fig. 3), we find, as expected from the simple cuts, that $C_{\text {cov }}$ (TSZ, KSZ) are not randomly distributed but they rather obey a general law. In particular, the highest covariance coefficients are clearly associated with temperature fluctuations arranged along a straight line in the $\left(C_{\text {cov }},(\delta T / T)_{\mathrm{KSZ}}\right)$ space. This behaviour exhibits, on a graphical basis, the tight physical relation between TSZ and KSZ effects as they are both due to the same galaxy clusters.

In a second step, we analyse the relation between the covariance coefficients $C_{\text {cov }}(\mathrm{TSZ}, \mathrm{KSZ}+\mathrm{CMB})$, and the temperature fluctuations $(\delta T / T)_{\mathrm{KSZ}}$ (Fig. 4). Interestingly, the same kind of alignment in the $\left(C_{\mathrm{cov}},(\delta T / T)_{\mathrm{KSZ}}\right)$ space, observed for a pure KSZ signal, holds for the mixture of KSZ and primary $\mathrm{CMB}$ anisotropies (note that $\mathrm{KSZ}$ signal is almost one order of magnitude smaller than the primary CMB). In this case and especially for the highest covariance coefficients, the spatial distribution of the data points (i.e. their overall alignment) also exhibits the relation between the covariance and the underlying KSZ temperature fluctuations. The bundle of points centred around zero corresponds mainly to the noise associated with the fortuitous and random correlations between the TSZ and pure $\mathrm{CMB}$, already observed in the cuts. We can definitely think of the relation $(\delta T / T)_{\mathrm{KSZ}}=f\left(C_{\mathrm{cov}}(\mathrm{TSZ}, \mathrm{KSZ}+\mathrm{CMB})\right)$ as an empirical statistical indicator of the presence of KSZ secondary anisotropies in the temperature fluctuation map. An obvious characterisation consists of expressing this relation in terms of a linear fit with non-zero intercept of the form:

$\left(\frac{\delta T}{T}\right)_{\mathrm{KSZ}}=\alpha C_{\mathrm{cov}}(\mathrm{TSZ}, \mathrm{KSZ}+\mathrm{CMB})+\beta$,

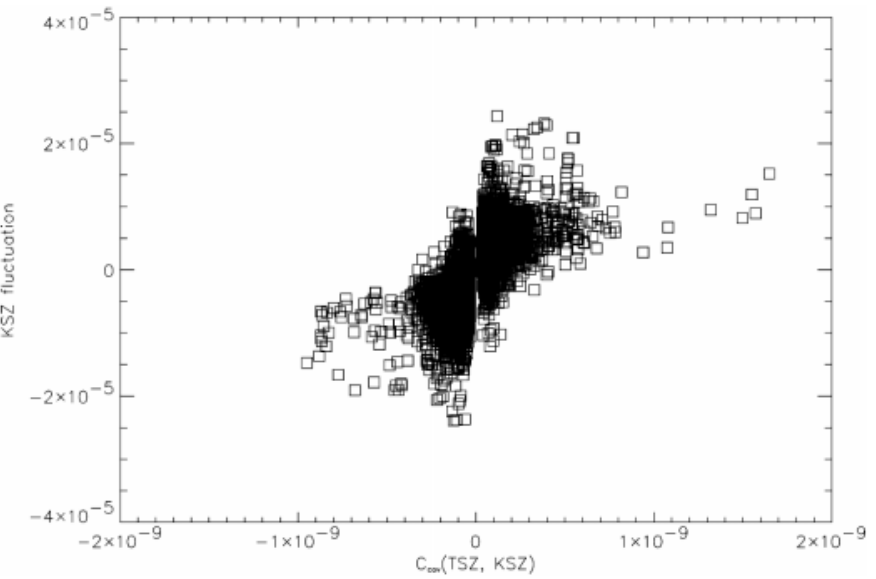

Fig. 3. For the 15 simulated maps, covariance coefficients, computed in a $2 \times 2$ pixel window, between the TSZ and pure KSZ signals (above $3 \sigma$ threshold) as a function of $(\delta T / T)_{\mathrm{KsZ}}$.

where $\alpha$ and $\beta$ are the two parameters of the law which can be obtained by fitting the data points corresponding to the highest covariance coefficients. The values of these parameters therefore depend on the amplitude of both the temperature fluctuations and TSZ distortions. Both are directly linked to the mass and abundance of galaxy clusters, to the cluster velocity distribution and to the primary CMB anisotropies. They hence depend on the underlying cosmological model which also rules, among other things, the number of fortuitous and random correlations between TSZ and pure CMB. The values of $\alpha$ and $\beta$ can therefore only be drawn for a particular cosmological model and instrumental configuration. It is however remarkable to notice that the relation between the covariance coefficients and the temperature fluctuations exhibits the buried KSZ signal despite the fact that it has the same spectral signature as the primary CMB anisotropies, and is one order of magnitude smaller. The covariance coefficients seem to trace very well the temperature fluctuations due to the KSZ effect buried in the CMB. This is perfectly illustrated, in Fig. 5 where we plot the covariance coefficients $C_{\text {cov }}(\mathrm{TSZ}, \mathrm{KSZ}+\mathrm{CMB})$ against $C_{\text {cov }}(\mathrm{TSZ}, \mathrm{KSZ})$, by the alignment of the points along a line of slope unity. Consequently, the covariance product between TSZ and CMB signals can be viewed as a criterion characterising the KSZ effect which could ease the separation between these spectrally indistinguishable signals.

When we compute the covariance factor in a larger window $(4 \times 4$ pixels, i.e., 6 arcmin aside), the results remain similar with the same general trend (Fig. 4, lower panel). It indicates that the difference in power spectrum between the KSZ effect and the primary CMB is important enough to allows us to detect the SZ effect, and hence the galaxy clusters.

\subsection{Statistical distribution of the covariance coefficients}

As exhibited above, the covariance seems a good statistical indicator of the presence of KSZ fluctuations in the signal through the linear relation between the covariance coefficient and $(\delta T / T)_{\mathrm{KSZ}}$. We now investigate the characteristics 

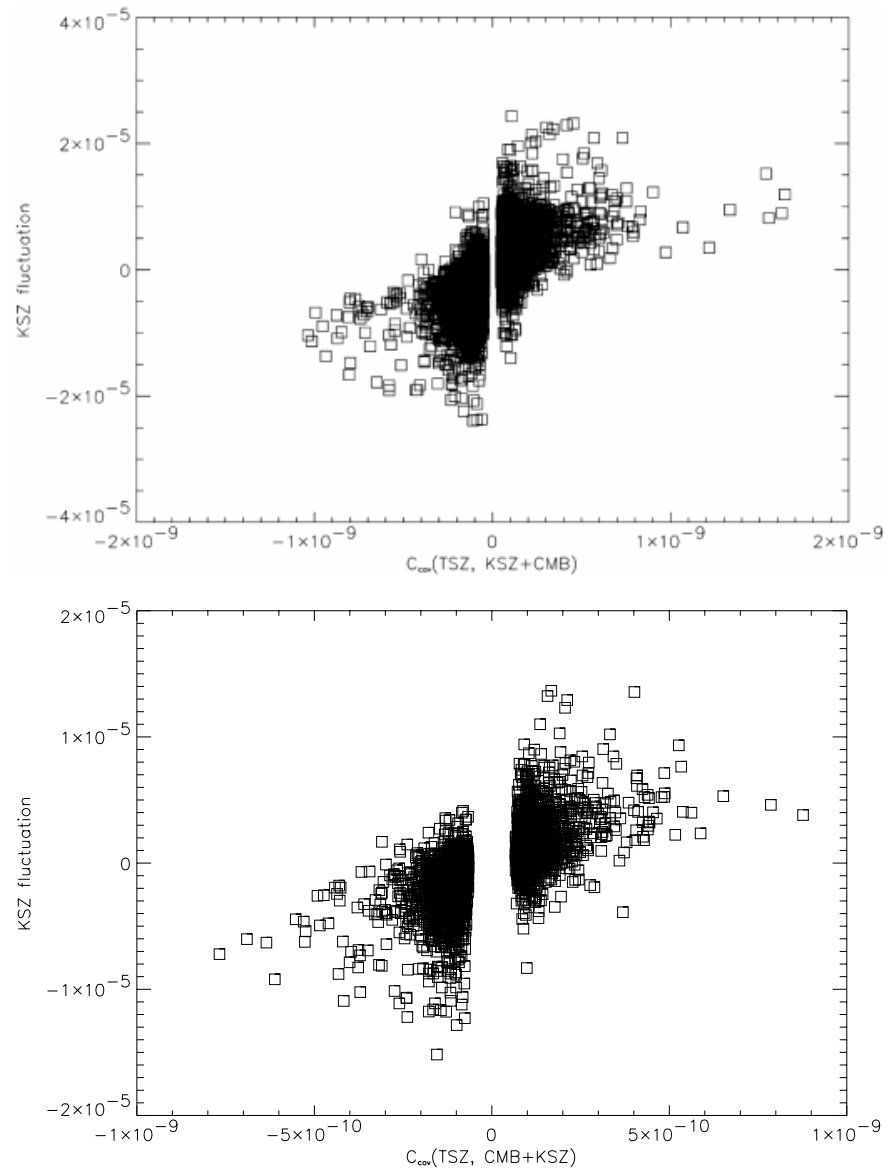

Fig. 4. Upper panel: for the 15 simulated maps, covariance coefficients, in a $2 \times 2$ pixel window, between the TSZ and KSZ + CMB signals (above $3 \sigma)$ as a function of $(\delta T / T)_{\mathrm{KsZ}}$. Lower panel: for the same maps, covariance coefficient (above $3 \sigma$ ) computed in a $4 \times 4$ pixel window between the TSZ and KSZ $+\mathrm{CMB}$ as a function of $(\delta T / T)_{\mathrm{KsZ}}$.

Table 1. The standard deviation of the covariance coefficients $(2 \times$ 2 pixel window) $\operatorname{Stddev}\left(C_{\text {cov }}\right)$, the mean skewness $\overline{S_{\text {cov }}}$, and its standard deviation $\sigma_{\mathrm{S}}$, for the covariance coefficients of the 15 maps.

\begin{tabular}{cccc}
\hline \hline & Stddev $\left(C_{\text {cov }}\right)$ & $\overline{S_{\text {cov }}}$ & $\sigma_{\mathrm{S}}$ \\
\hline $\mathrm{KSZ}$ & $1.78 \times 10^{-11}$ & 0.35 & 6.22 \\
$\mathrm{CMB}$ & $1.17 \times 10^{-11}$ & -0.01 & 0.18 \\
$\mathrm{KSZ}+\mathrm{CMB}$ & $2.10 \times 10^{-11}$ & 0.47 & 4.35 \\
\hline
\end{tabular}

of the distribution of covariance coefficients for each of the 15 maps. We notice again that the covariance coefficients have larger values when there is a KSZ contribution in the temperature fluctuation signal (i.e., pure $\mathrm{KSZ}$ or $\mathrm{KSZ}+\mathrm{CMB}$ ). This is exhibited by the standard deviation of the covariance coefficients for each map. We compute the mean standard deviation (over the 15 maps) (Table 1, first column) for the coefficients $C_{\text {cov }}(\mathrm{TSZ}, \mathrm{KSZ}), C_{\text {cov }}(\mathrm{TSZ}, \mathrm{KSZ}+\mathrm{CMB})$ and $C_{\text {cov }}(\mathrm{TSZ}, \mathrm{CMB})$. The standard deviation in the latter case is smaller than in the previous two. As expected, the temperature fluctuation map containing a KSZ contribution is obviously more "correlated" with the TSZ than an independent signal (primary CMB in our case). However, all three standard

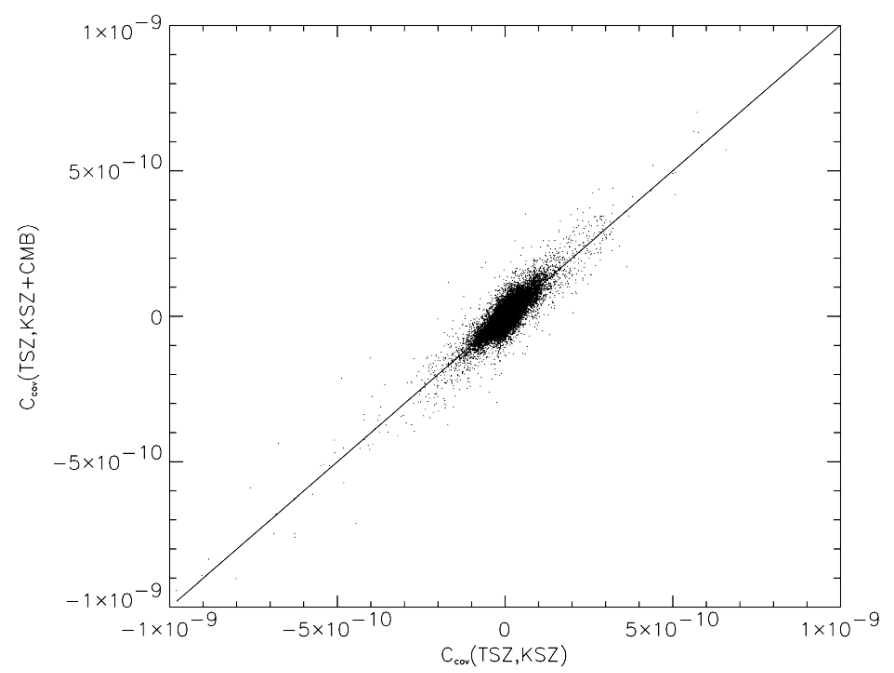

Fig. 5. Covariance coefficient between the TSZ and KSZ + CMB $C_{\text {cov }}(\mathrm{TSZ}, \mathrm{KSZ}+\mathrm{CMB})$ as a function of $C_{\text {cov }}(\mathrm{TSZ}, \mathrm{KSZ})(2 \times 2$ pixel window).

deviations remain rather close to each other with the ratio of Stddev (TSZ, KSZ+CMB) to Stddev (TSZ, CMB) equals 1.8, and the ratio of Stddev (TSZ, KSZ) to Stddev (TSZ, CMB) equals 1.5. In practice, there is no way to distinguish, through the standard deviation of the covariance coefficients, between the case where the KSZ fluctuations contribute to the signal and the case where they do not.

We therefore turn to the third moment of the distribution of the covariance coefficients, namely the skewness (Table 1, second column). We compute the skewness denoted $S_{\text {cov }}(T S Z, i)$ where $i$ stands for our three signals. For $i=\mathrm{KSZ}$, the skewness of each map ranges between -7.7 and 12.9 with a mean skewness $\overline{S_{\mathrm{cov}}}=0.35$ and a standard deviation $\sigma_{\mathrm{S}}=6.2$. For the covariance between TSZ and KSZ+CMB, we find the skewnesses ranging between -4.6 and 10.5 with mean and standard deviation respectively equal to 0.47 and 4.35 . If we now compute the same quantities for the covariance between TSZ and the pure primary CMB signal, we find that the skewness has smaller values. It ranges between -0.37 and 0.29 and has a mean of -0.01 and a standard deviation of 0.18 . The numbers we obtain for the skewness in each case shows that the KSZ and KSZ+CMB signals behave in similar ways. The values of the skewness are large as expressed by the standard deviation. Whereas for the pure primary CMB map, the skewness is always small. High values for the skewness are associated with highly non-symmetric distributions of covariance coefficients. This feature can already be derived from Fig. 4. It indicates an excess of signal due to the presence of positive KSZ temperature fluctuations (in our case), i.e., galaxy clusters with negative peculiar velocities. In our simulations, this excess of negative velocities is related to the statistical realisations. However, we expect that the galaxy clusters will exhibit large scale coherent motions. On small pieces of the sky, these coherent bulk motions will translate, in the same manner, into large values of the skewness. Therefore, similarly to the linear relation between $(\delta T / T)_{\mathrm{SZ}}$ and $C_{\mathrm{cov}}$, the skewness of the covariance coefficients seems a promising blind statistical indicator of the presence of 
KSZ anisotropies as soon as the skewness is large enough (typically unity).

\section{Discussion and conclusion}

In the new general context of CMB observation, the component separation is a key issue for the precision needed to accurately estimate the cosmological parameters. It is also a necessity to reach the accuracy needed for the numerous scientific projects that are aimed with the foreground emissions and secondary contributions. The component separation usually takes advantage of the multi-frequency observations that allow us to distinguish between the various signals through their spectral signatures; as it is the case for the TSZ effect for example.

However, some astrophysical contributions have the same spectral signature as the primary CMB anisotropies. They can therefore not be separated from it and they remain as an additional buried signal, source of an additional and systematic error. In particular, the KSZ effect falls into this category of signals. It represents a contaminating component that should be subtracted from the primary anisotropies, and in addition, it is a necessary information to estimate the cluster peculiar velocities. The KSZ effect has a small amplitude as compared with the primary CMB fluctuations $(\simeq 10 \%)$ except at angular scales of about a few arcminutes where it becomes of the same order of, or exceeds, the primary signal.

The present study aims at finding statistical indicators that hint to the presence of KSZ fluctuations mixed to the CMB signal. In our empirical approach, we only assume that we are able to recover a TSZ map, easily separated due to its spectral signature, and a temperature map (containing KSZ fluctuations). We show that the covariance between the TSZ map and the temperature fluctuation map presents interesting and promising properties that we use to blindly exhibit the presence of secondary temperature fluctuations due to KSZ effect mixed to primary CMB. The skewness, i.e. third moment of the distribution of the covariance coefficients, can be considered as a blind statistical indicator of the presence of KSZ anisotropies, when it is roughly above unity. It may indicate coherent motions. The linear relation between the covariance coefficients and the temperature fluctuations, when it includes KSZ anisotropies, seems a second powerful blind statistical indicator of the KSZ effect. It relates the amplitude of the covariance coefficients with the secondary anisotropies. We have checked that the linear relation is preserved when we simulate the observing process, i.e. taking into account the beam dilution and the instrumental noise in the case of Planck surveyor.

The statistical indicators of the KSZ additional signal we propose here can by no means stand for a proper component separation that would allow us to subtract the KSZ fluctuations from the temperature map and obtain accurate measurements. Nevertheless, these indicators can be viewed as a promising way of approaching the separation using directly the maps. In the context of the present and near future small scale multifrequency CMB experiments, the indications of the KSZ contribution from our simple statistical tools could at least motivate us to consider KSZ as an additional error, which first order amplitude may be derived from theoretical predictions.

Acknowledgements. The authors wish to thank A. Jones for his comments and J.-L. Puget for interesting discussions. We also thank an anonymous referee for her or his remarks.

\section{References}

Aghanim, N., \& Forni, O. 1999, A\&A, 347, 409

Aghanim, N., Górski, K. M., \& Puget, J. L. 2001, A\&A, 374, 1

Birkinshaw, M. 1999, Phys. Rep., 310, 97

Bouchet, F. R., \& Gispert, R. 1999, New Astron., 4, 443

de Bernardis, P., Ade, P. A. R., Bock, J. J., et al. 2002, ApJ, 564, 559

Hobson, M. P., Jones, A. W., Lasenby, A. N., \& Bouchet, F. R. 1998, MNRAS, 300, 1

Jewell, J. 2001, ApJ, 557, 700

Pryke, C., Halverson, N. W., Leitch, E. M., et al. 2002, ApJ, 568, 746

Snoussi, H., Patanchon, J. F., Macias-Perez, J. F., Mohammed-Djafari, A., \& Delabrouille, J. 2001, in Proceedings of the MAXENT 2001 international workshop

Sunyaev, R. A., \& Zel'dovich, I. B. 1980, ARA\&A, 18, 537

Wang, X., Tegmark, M., \& Zaldarriaga, M. 2002, Phys. Rev. D, 65, 123001 